\title{
Automatic 3D tracking of cardiac material markers using slice-following and harmonic-phase MRI ${ }^{\text {tr }}$ \\ Smita Sampath*, Jerry L. Prince \\ Department of Electrical and Computer Engineering, Johns Hopkins University, Baltimore, MD 21218, USA \\ Received 7 June 2006; accepted 15 September 2006
}

\begin{abstract}
A method to track a grid of cardiac material points in three dimensions using slice-following (SF) tagged magnetic resonance imaging (MRI) and harmonic-phase MRI is presented. A three-dimensional grid of material points on the lines of intersections of short-axis (SA) and long-axis (LA) planes is automatically tracked by combining two-dimensional pathlines that are computed on both SA and LA image planes. This process yields the true three-dimensional motion of points originating on the image plane intersections. Experimental data from normal volunteers, each obtained in four short breath-holds using the SF harmonic phase MRI pulse sequence, is presented. A validation of twodimensional in-plane tracks using this pulse sequence on a moving phantom is also presented.
\end{abstract}

(C) 2007 Elsevier Inc. All rights reserved.

Keywords: Magnetic resonance tagging; HARP; Slice-following; Cardiac motion; Cardiac strain

\section{Introduction}

Visualizing and quantifying cardiac motion are important steps in the diagnosis and management of heart disease. Current practice is largely limited to two-dimensional analyses (2-D), despite the availability of relatively straightforward protocols for three-dimensional (3-D) imaging. In magnetic resonance imaging (MRI), for example, tagging $[1,2]$, phase contrast [3-5] and stimulated echo [6,7] methods are capable of detailed 2-D imaging and analysis. Three-dimensional visualization and quantification, however, is not readily available, largely because of a lack of fast, automatic image acquisition and processing approaches. This deficiency is addressed in this article.

Routine, 3-D visualization and quantification of cardiac motion should be clinically useful. For example, it has been

Jerry L. Prince is a founder of and owns stock in Diagnosoft, a company which seeks to license the HARP technology. The terms of this arrangement are being managed by the Johns Hopkins University in accordance with its conflict of interest policies.

th This work was supported by a National Heart, Lung, and Blood Institute grant (R01HL47405; PI Jerry Prince).

* Corresponding author. Laboratory of Cardiac Energetics, National Institutes of Health, National Heart, Lung, And Blood Institute, Bethesda, MD 20892-1061, USA. Tel.: +1 3014961159.

E-mail address: sampaths@mail.nih.gov (S. Sampath). shown that in hypertrophic cardiomyopathy, torsion in the left ventricle (LV) is reduced, with significantly less rotation in the posterior region of the equatorial and apical planes [8]. Ischemia and infarction are known to affect the motion of the endocardial wall [9], to correlate well with wall thickening [10], and to reduce regional rotational and translational motion $[11,12]$. Patterns of normal basal and apical rotations - a "wringing" action - of the LV have been studied [13], and alterations in these rotational patterns in hypertrophic cardiomyopathy $[14,15]$ after myocardial infarction [11] have been reported. Mechanical activation of the paced heart has been imaged and analyzed to reveal significant asynchrony and heterogeneous contraction, depending on the location of the pacemaker lead [16].

Magnetic resonance imaging has been at the core of the most sophisticated and detailed analyses of 3-D myocardial motion. Data derived from magnetic resonance (MR) tagging, phase-contrast velocity images and stimulated echo images have all been used to infer global 3-D motion properties of the heart, including strain and strain rates, displacement, velocity, streamlines and pathlines [17]. But there are still significant challenges. For example, tracking of dense 3-D displacement inevitably requires long imaging times $[3,7,18,19]$, manually intensive postprocessing $[18,20,21]$ and/or interpolation techniques [22-24]. 
In this article, we back off from the goal of dense tracking and propose a new method for automatic tracking of a sparse collection of myocardial points. The method, which we call slice-following harmonic phase (SF-HARP) imaging, applies 2-D harmonic phase (HARP) analysis methods $[25,26]$ to complementary spatial modulation of magnetization (CSPAMM)-tagged MR images that have been acquired using the slice-following (SF) technique $[27,28]$. Acquiring multiple (orthogonal) images permits the 3-D tracking of points at the intersections of these image planes. We use this approach as the basis for computing global quantities such as myocardial rotation and twist, but it could also be used for computing regional strain by acquiring multiple image planes in a small region.

In the following, we briefly describe both SF with CSPAMM (SF-CSPAMM) and HARP (which are established techniques), and then describe SF-HARP. The performance of SF-HARP is then demonstrated by presenting results on both normal volunteers and a moving phantom.

\section{Theory}

\subsection{Slice-following and 2-D HARP}

The SF-CSPAMM technique $[28,29]$ was introduced to take through-plane motion into consideration while imaging spatial modulation of magnetization (SPAMM)-tagged slices [2]. In SF-CSPAMM, a thin slice is tagged at the initial time frame while imaging a large slab that always encompasses the moving tagged slice even at later time
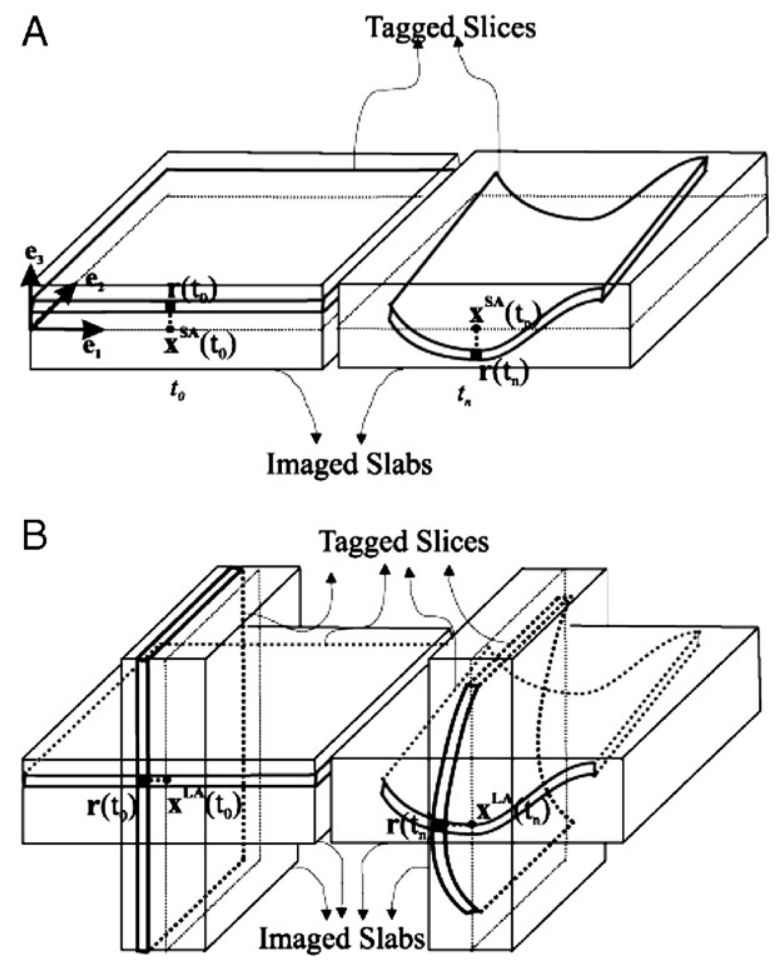

Fig. 1. Tagged slices are encompassed by the imaged slices in SF with CSPAMM. (A) Single imaged slice at time frames $t_{0}$ and $t_{\mathrm{n}}$. (B) Two orthogonal imaged slices at the two time frames. frames [see Fig. 1(A)]. Two image sequences with complementarily signed (1-1 SPAMM) tagging modulations are acquired at each slice location: the $A$ sequence uses a $\left[+90^{\circ},+90^{\circ}\right]$ tagging pulse, and the $B$ sequence uses a $\left[+90^{\circ},-90^{\circ}\right]$ tagging pulse.

Consider two SF-CSPAMM image sequences, one acquired in the short-axis (SA) plane and the other in the long-axis (LA) plane within the heart. It is useful to define an anatomic coordinate system for these two orthogonal planes. Let $\left(\mathbf{e}_{1}, \mathbf{e}_{2}, \mathbf{e}_{3}\right)$ be a 3-D orthonormal frame, and let $\left(\mathbf{e}_{1}, \mathbf{e}_{2}\right)$ be coordinate directions in the SA plane and $\left(\mathbf{e}_{2}, \mathbf{e}_{3}\right)$ be coordinate directions in the LA plane. Suppose $\mathbf{x}=\left(x_{1}, x_{2}\right)$ is a 2-D vector representing a position within a given image plane. In the SA plane, $x_{1}$ gives the position in the $\mathbf{e}_{1}$ direction and $x_{2}$ gives the position in the $\mathbf{e}_{2}$ direction. On the other hand, in the LA plane, the first and second elements of $\mathbf{x}$ correspond to the $\mathbf{e}_{2}$ and $\mathbf{e}_{3}$ directions, respectively. All positions are measured relative to an origin, which is taken to be somewhere on the intersection of the two planes. It is observed that the coordinate direction $\mathbf{e}_{2}$ is common to the two coordinate systems, and because of our choice of origin, $\mathbf{e}_{2}$ defines the direction of the line created by the intersection of the two image planes.

The spatial distribution of the longitudinal magnetization at any time $t$ for the images $A$ and $B$ (in either image plane) is given by

$$
\begin{aligned}
A(\mathbf{x}, t)= & {\left[M_{0}(\mathbf{x}) \cos [\varphi(\mathbf{x}, t)] e^{-t / T 1}\right.} \\
& \left.+M_{0}(\mathbf{x})\left(1-e^{-t / T 1}\right)\right] f[\cos (\alpha) ; t]
\end{aligned}
$$

and

$$
\begin{aligned}
B(\mathbf{x}, t)= & {\left[M_{0}(\mathbf{x}) \cos [\varphi(\mathbf{x}, t)-\pi] e^{-t / T 1}\right.} \\
& \left.+M_{0}(\mathbf{x})\left(1-e^{-t / T 1}\right)\right] f[\cos (\alpha) ; t]
\end{aligned}
$$

where $M_{0}(\mathbf{x})$ is the initial longitudinal magnetization, $f[\cos (\alpha) ; t]$ is a function of the imaging flip angles $\alpha$ and $\cos [\phi(\mathbf{x}, t)]$ and $\cos [\phi(\mathbf{x}, t)-\pi]$ are the modulated motion-related tagging functions. The complex subtraction $A-B$ prescribed by CSPAMM [27] yields a series of SFCSPAMM images given by

$$
\begin{aligned}
& I_{\mathrm{SF}-\mathrm{CSPAMM}}(\mathbf{x}, t) \\
& \quad=2 M_{0}(\mathbf{x}) e^{-t / T 1} \cos [\varphi(\mathbf{x}, t)] f[\cos (\alpha) ; t] .
\end{aligned}
$$

Slice-following guarantees that this signal arises from the tagged slice itself rather than from tissues that might have entered the image slab as the result of through-plane motion.

The Fourier transform of each image in Eq. (3) has two harmonic peaks. Although cardiac motion causes local shifts in the frequency and/or broadening of these harmonic peaks during the cardiac cycle, the motion information is largely contained within small regions in $\mathrm{k}$-space surrounding these harmonic peaks. HARP techniques [25] typically use a band-pass filter to isolate regions of interest around one of 

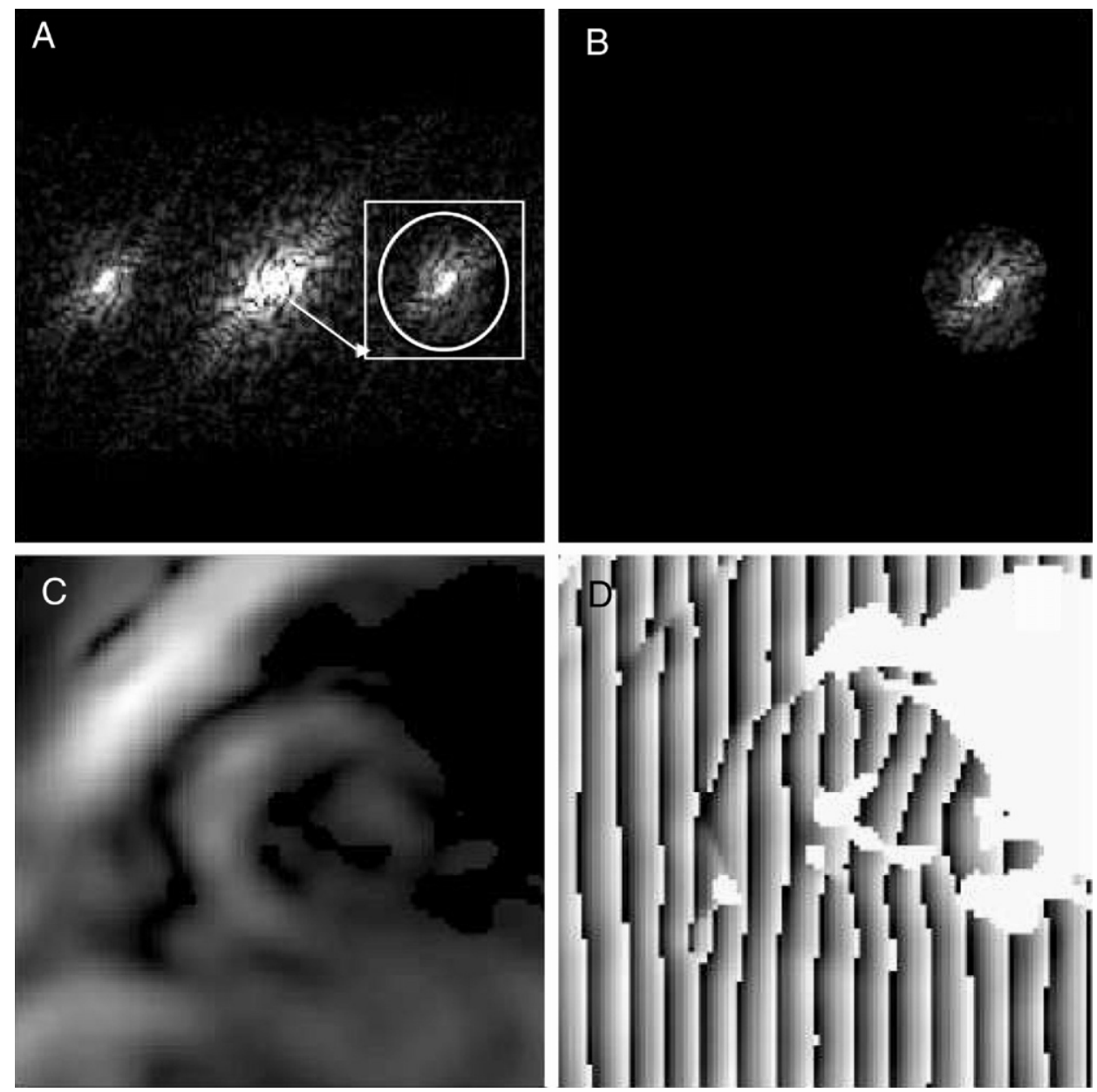

Fig. 2. (A) The k-space of a 1-1 SPAMM tagged image, the acquisition window (white box), the band-pass filter (yellow circle); (B) isolated harmonic peak; (C) harmonic magnitude image and (D) harmonic phase image.

these peaks (see Fig. 2) to compute a sequence of (complexvalued) harmonic images given by

$I(\mathbf{x}, t)=2 M_{0}(\mathbf{x}) e^{-t / T 1} e^{j \varphi(\mathbf{x}, t)} f[\cos (\alpha) ; t]$.

The phase $\phi(\mathbf{x}, t)$ of these images — the so-called harmonic phase images - are directly related to the motion of the tagged slice in the direction perpendicular to the tag lines. Two-dimensional pathlines of any point in the image plane can be computed by tracking the 2-D harmonic phase values obtained from horizontal and vertical tagged images $[25,26]$.

\subsection{SF-HARP material point tracking}

Let the time of tag application be given by $t_{0}$. Consider a material point located at a $3-\mathrm{D}$ position $\mathbf{r}\left(t_{0}\right)=r_{1}$ $\left(t_{0}\right) \mathbf{e}_{1}+r_{2}\left(t_{0}\right) \mathbf{e}_{2}+r_{3}\left(t_{0}\right) \mathbf{e}_{3}$ on a SA tagged slice, as illustrated in Fig. 1(A). At a later time $t_{n}$ the point $r\left(t_{\mathrm{n}}\right)$ lies on the deformed SA tagged slice. Because of SF, the image value corresponding to $\mathbf{r}\left(t_{n}\right)$ is recorded at position $\mathbf{x}^{\mathrm{SA}}=\left[r_{1}\left(t_{n}\right)\right.$, $r_{2}\left(t_{n}\right)$ ], an orthogonal projection of $\mathbf{r}\left(t_{n}\right)$ onto the central cross-sectional plane of the SA image slab.

Now, consider two orthogonal tagged slices, as shown in Fig. 1(B), and assume that point $\mathbf{r}\left(t_{0}\right)$ lies on the line of SA and LA tagged slice intersection. At the later time $t_{n}, \mathbf{r}\left(t_{n}\right)$ will also lie on the deformed LA tagged slice, and its imaged coordinate will be $\mathbf{x}^{\mathrm{LA}}=\left[r_{2}\left(t_{n}\right), r_{3}\left(t_{n}\right)\right]$, an orthogonal projection of $\mathbf{r}\left(t_{n}\right)$ onto the central cross-sectional plane of the LA image slab [see Fig. 1(B)]. Thus, over time, the point moves in three dimensions due to the motion of the heart, creating the 3-D pathline $\mathbf{r}(t)$. The point must remain on both SA and LA deformed tagged slices and is therefore imaged twice by orthogonal projections, yielding two 2-D tracks,

$\mathbf{x}^{\mathrm{SA}}(t)=\left(r_{1}(t), r_{2}(t)\right)$

and

$\mathbf{x}^{\mathrm{LA}}(t)=\left(r_{2}(t), r_{3}(t)\right)$.

If these tracks can be determined, then the 3-D motion of $\mathbf{r}\left(t_{0}\right)$ is easily found, as described below.

2-D HARP tracking uses the principle that the HARP phase of a point remains constant as it moves around in the plane [25]. This principle applies equally well to the projection of a 3-D point, as is the case here. Fig. 3 shows SF-HARP images for a pair of such orthogonal slices 

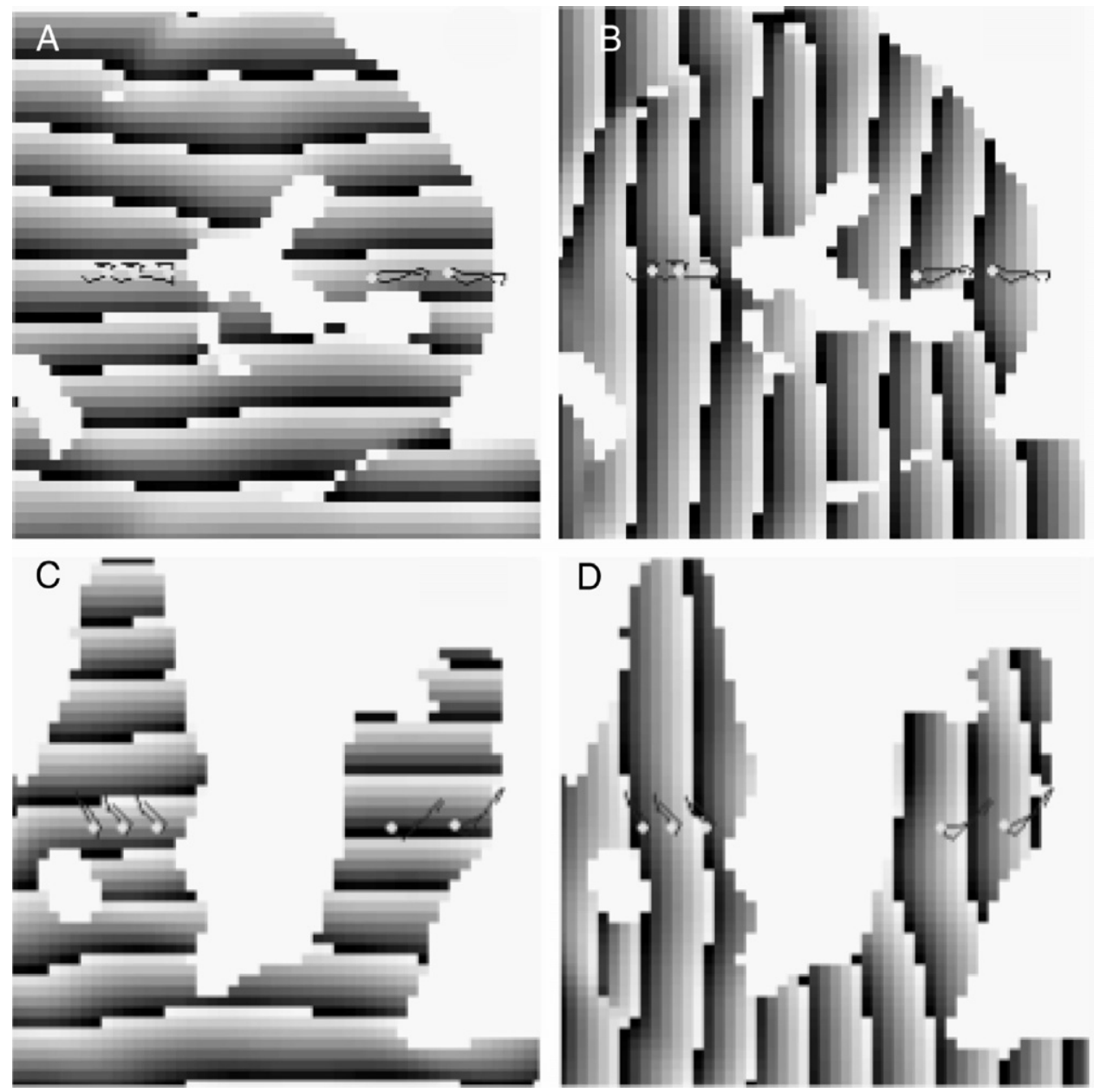

Fig. 3. Short- and long-axis HARP images at an end-systolic time frame obtained using the SF-HARP MRI pulse sequence with tags oriented in the horizontal [(A) and (C)] and vertical [(B) and (D)] directions, respectively. The black trajectories denote the 2-D path lines of five points selected on the line of intersection of the two image planes. The white dots denote the position of the points in the end-systolic time frame displayed here.

acquired at an end-systolic time frame with tags oriented along the horizontal and vertical directions. Let the vector $\phi(\mathbf{x}, t)=\left[\phi^{h}, \phi^{v}\right]$ describe the horizontal and vertical harmonic phase images for either a SA or LA orientation. Then 2-D HARP tracking finds the sequence of points $\mathbf{x}\left(t_{n}\right)$ such that $\phi\left(\mathbf{x}\left(t_{n}\right), t_{n}\right)=\phi\left(\mathbf{x}\left(t_{0}\right), t_{0}\right)$, for image frames $n=1, \ldots, N$. Therefore, 2-D HARP tracking can be used to find the two 2-D tracks $\mathbf{x}^{\mathrm{SA}}\left(t_{n}\right)$ and $\mathbf{x}^{\mathrm{LA}}\left(t_{n}\right)$ within the SA and LA image planes over time frames $n=0, \ldots, N$. Fig. 3 shows several such pathlines for a pair of orthogonal slices acquired at an end-systolic time frame with tags oriented along the horizontal and vertical directions. The gray lines depict the 2-D tracked trajectories of five points overlaid on the SF-HARP images. The white dots indicate the position of the material points at the end-systolic time frame in view.

Given two estimated 2-D tracks,

$\mathbf{x}^{S A}\left(t_{n}\right)=\left[a\left(t_{n}\right), b\left(t_{n}\right)\right]$

and

$\mathbf{x}^{L A}\left(t_{n}\right)=\left[c\left(t_{n}\right), d\left(t_{n}\right)\right]$,

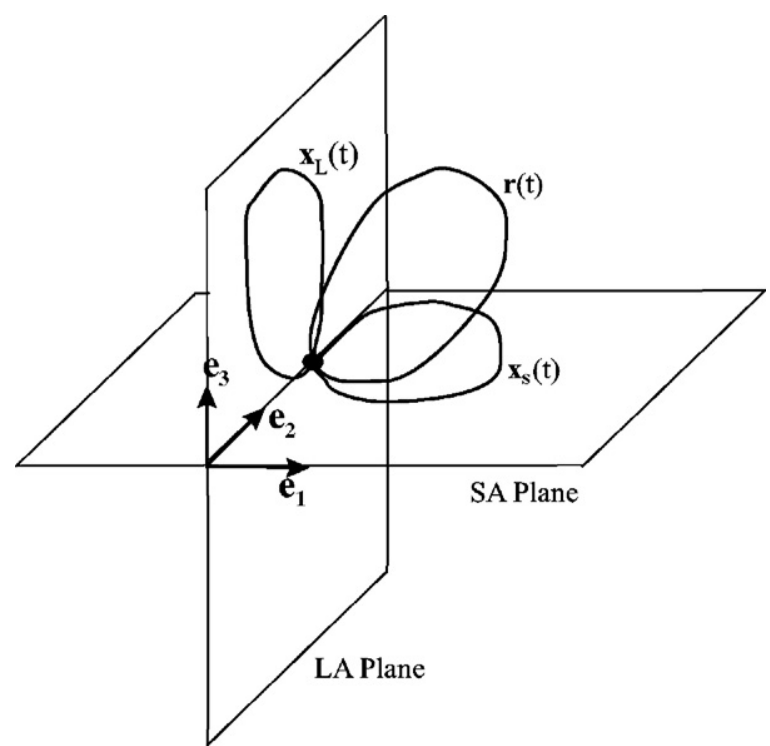

Fig. 4. Short- and long-axis image planes with coordinate systems and HARP tracks on each plane. The 3-D track $\mathbf{r}(t)$ is deduced from the planar tracks. 
the actual 3-D trajectory of the point $\mathbf{r}\left(t_{0}\right)$ is determined by

$\mathbf{r}\left(t_{n}\right)=a\left(t_{n}\right) \mathbf{e}_{1}+\frac{b\left(t_{n}\right)+c\left(t_{n}\right)}{2} \mathbf{e}_{2}+d\left(t_{n}\right) \mathbf{e}_{3}, n=0, \ldots, N$.

An average of the two coordinate functions determined for the $\mathbf{e}_{2}$ direction is used to reduce noise. This scenario is depicted in Fig. 4.

\section{Methods}

\subsection{SF-HARP pulse sequence}

Our goal was to acquire enough image planes to characterize global 3-D left ventricular motion in a small number of breath-holds. Accordingly, we designed the SFHARP MRI pulse sequence for a 1.5T GE Signa CV/I wholebody MR system by starting with the sequential fast gradient echo HARP MRI pulse sequence [30], adding modifications to include both CSPAMM and SF features. A 1-1 SPAMM tagging scheme comprising two $90^{\circ}$ tagging RF pulses was used, the first pulse being slice-selective and the second being hard, and the two pulses were separated by a tagging gradient. For a given slice and a given tag direction, thin slices were selectively tagged, and a sequence of harmonic images with thicker slices, centered at the same location as the tagged slice, were subsequently imaged in a single heartbeat.

Harmonic images were directly acquired by restricting the $\mathrm{k}$-space acquisition to a $32 \times 32$ square matrix size centered on a harmonic peak of interest as shown in Fig. 2(A). A four-shot, bottom-up, echo-planar imaging scheme was used to acquire the data with the following imaging parameters: tagged slice thickness, $8 \mathrm{~mm}$; tag spacing, $8 \mathrm{~mm}$; image slab thickness, $30 \mathrm{~mm}$; receiver bandwidth, $\pm 62.5 \mathrm{kHz}$; TR/TE, $12.25 / 2 \mathrm{~ms}$ and an incrementing train of imaging flip angles with an optimized final flip angle of $15^{\circ}$ [28].

Four separate sequences of harmonic images were obtained for each slice, and each sequence was acquired in a single heartbeat comprising 10-15 time frames with a temporal resolution of $48 \mathrm{~ms}$. A view-sharing scheme (i.e., a sliding window combination of $\mathrm{k}$-space acquisitions) could be used to produce an image every $12 \mathrm{~ms}$. The first sequence of images was acquired with regular SPAMM tags applied in the horizontal direction, while the second sequence of images was acquired with the tags oriented in the vertical direction. Similarly, the third and fourth sequence of images were acquired with complimentarily signed SPAMM tags oriented in the horizontal and vertical directions respectively. A fourchannel cardiac phased-array coil was used to receive the MR signal. For each coil, a smooth bandpass filter operated on every k-space image to further isolate the harmonic peak [see Fig. 2(A)], which was zero-padded to a matrix size of $256 \times 256$ [see Fig. 2(B)] and reconstructed using an inverse Fourier transform to obtain a harmonic image.
The four harmonic image sequences can be mathematically expressed as:

$I_{i}^{a h}(\mathbf{x}, t)=D_{0}(\mathbf{x}, t)+D(\mathbf{x}, t) e^{j \varphi^{h}(\mathbf{x}, t)} M_{i}(\mathbf{x}) e^{j \theta_{i}(\mathbf{x})}$,
$I_{i}^{a v}(\mathbf{x}, t)=D_{0}(\mathbf{x}, t)+D(\mathbf{x}, t) e^{j \varphi^{v}(\mathbf{x}, t)} M_{i}(\mathbf{x}) e^{j \theta_{i}(\mathbf{x})}$,
$I_{i}^{b h}(\mathbf{x}, t)=D_{0}(\mathbf{x}, t)-D(\mathbf{x}, t) e^{j \varphi^{h}(\mathbf{x}, t)} M_{i}(\mathbf{x}) e^{j \theta_{i}(\mathbf{x})}$

and

$I_{i}^{b v}(\mathbf{x}, t)=D_{0}(\mathbf{x}, t)-D(\mathbf{x}, t) e^{j \varphi^{v}(\mathbf{x}, t)} M_{i}(\mathbf{x}) e^{j \theta_{i}(\mathbf{x})}$,

where $I_{i}(\mathbf{x}, t)$ represents the image from coil $i$ at time frame $t$ and position $\mathbf{x}$. The superscripts $a$ and $b$ represent the SPAMM and CSPAMM images, respectively, while the superscripts $h$ and $v$ stand for horizontally and vertically tagged images, respectively. $D_{0}(\mathbf{x}, t)$ represents a signal from the DC peak at position $\mathbf{x}$ and time $t$ while $D(\mathbf{x}, t)$, $\phi^{h}(\mathbf{x}, t)$ and $\phi^{v}(\mathbf{x}, t)$ represent the magnitude and phases (horizontal and vertical) of the harmonic images at position $\mathbf{x}$ and time $t$. The last, time-independent, complex term in Eqs. (10)-(13) represents the complex sensitivity profile of the coil $i$. Subtracting the CSPAMM images from the SPAMM images yields

$I_{i}^{h}(\mathbf{x}, t)=2 \mathrm{D}(\mathbf{x}, t) e^{j \varphi^{h}(\mathbf{x}, t)} M_{i}(\mathbf{x}) e^{j \theta_{i}(\mathbf{x})}$

and

$I_{i}^{v}(\mathbf{x}, t)=2 \mathrm{D}(\mathbf{x}, t) e^{j \varphi^{v}(\mathbf{x}, t)} M_{i}(\mathbf{x}) e^{j \theta_{i}(\mathbf{x})}$.

The multicoil magnitude data is then combined using a standard sum-of-squares procedure to give $D_{\text {comb }}^{h}(\mathbf{x}, t)$ and $D_{\text {comb }}^{v}(\mathbf{x}, t)$. To perform phase combination of the multicoil data, two sets of phase reference images are acquired for each slice. During these reference scans, the acquisition window is centered on the DC peak rather than the harmonic peak. The multicoil phase is then combined using an extension of the corrected phase difference method [31] to give $\phi_{\mathrm{comb}}^{h}(\mathbf{x}, t)$ and $\phi_{\mathrm{comb}}^{v}(\mathbf{x}, t)$. Using the combined magnitude and phase terms in Eqs. (10)-(13), the two sets of reconstructed SF harmonic images with orthogonally tagged images for any given slice can be expressed as

$I_{\text {comb }}^{h}(\mathbf{x}, t)=D_{\text {comb }}^{h}(\mathbf{x}, t) e^{j \varphi_{\text {comb }}^{h}(\mathbf{x}, t)}$

and

$I_{\text {comb }}^{v}(\mathbf{x}, t)=D_{\text {comb }}^{v}(\mathbf{x}, t) e^{j \varphi_{\text {comb }}^{v}(\mathbf{x}, t)}$.

The images in Eqs. (16) and (17) represent reconstructions of the tag slice alone; signal contributions from the remainder of the image slice have been subtracted out. The SF harmonic magnitude image $D_{\text {comb }}^{v}(\mathbf{x}, t)$ and the motiondependent wrapped SF-HARP image $\phi_{\text {comb }}^{v}(\mathbf{x}, t)$ for an endsystolic SA left-ventricular image are shown in Fig. 2(C) and (D), respectively.

Multiple slices are acquired sequentially using this pulse sequence, where the complete data for each slice is acquired 

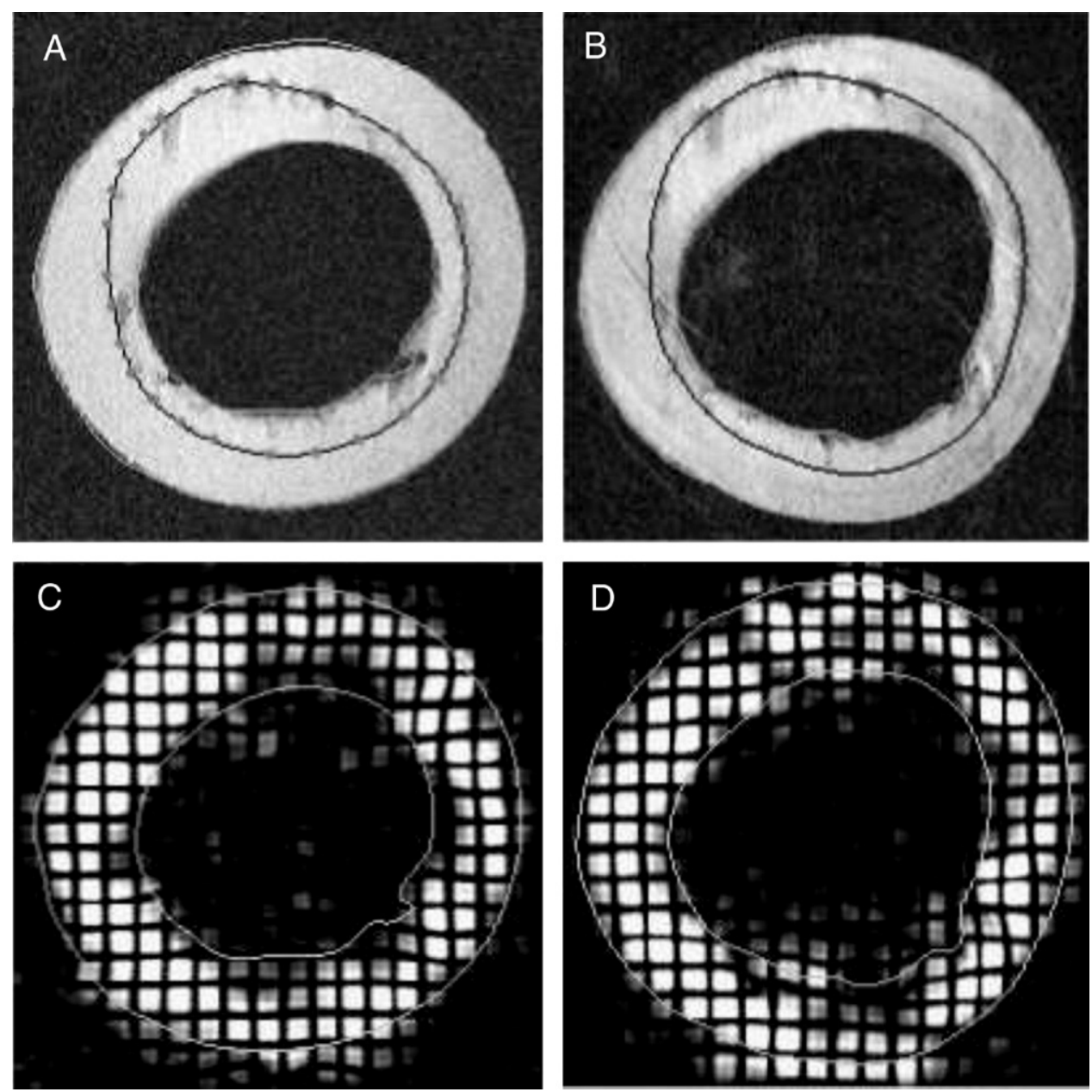

Fig. 5. Representative images from the phantom experiment. Manually generated mesh boundaries (black lines) superimposed on the high-resolution images at (A) $391 \mathrm{~ms}$ (Mesh1) and (B) $1292 \mathrm{~ms}$ (Mesh2). Black dots on the black line in (A) represent a selected set of thirty points on Mesh1. Tagged images at time frames (C) $394 \mathrm{~ms}$ and (D) $1296 \mathrm{~ms}$. White lines superimposed on all images represent inner and outer boundaries of the phantom slice.

one after the other. Each slice requires a six-heartbeat acquisition to obtain the reference images, the regular tagged harmonic images and the complementarily tagged harmonic images for the two orthogonal tag orientations. Thus, if $M$ slices are required, a total breath-held acquisition time of $6 M T$ is needed, where $T$ is the time required to acquire one slice with one tag orientation and one CSPAMM component.

\subsection{Experimental methods: phantom study}

\subsubsection{Phantom design}

A phantom was made of Dow Corning Sylgard 527 gel $(\mathrm{A}: \mathrm{B}=1: 1)$ and was in the shape of a cylindrical annulus with an inner diameter of $3^{\prime \prime}$ and an outer diameter of $5^{\prime \prime}$ [32]. The phantom motion was actuated using a purely pneumatic scheme. Four air cells were embedded in the hollow column of the cylindrical phantom so that each air cell was roughly responsible for inflating one quadrant of the cylinder. When the air cells were inflated using compressed air, the walls of the phantom were pushed outwards; when the air cells were deflated, they returned to their original positions. The motion generated was repeatable since the gel is a highly elastic material, yielding a rate of 44 cycles per minute (1363-ms cycle). The point of triggering coincided with the start of the deflation phase and was provided using a peripheral pulse device with the infrared sensor placed close to the moving phantom.

\subsubsection{Image acquisition}

Two kinds of data sets were acquired for a selected slice. First, a gated, segmented, sequential fast gradient-echo pulse sequence was used to acquire a sequence of 37 images with the following imaging parameters: field of view (FOV), $320 \mathrm{~mm}$; acquisition matrix, $512 \times 512$; receiver bandwidth, $\pm 31.25 \mathrm{kHz}$; phase encode lines per R-R, 8 and $\mathrm{TR}, 6200 \mathrm{~ms}$. The total acquisition time for this pulse sequence was approximately $9 \mathrm{~min}$. Second, the SF-HARP MRI pulse sequence described above was used to acquire six sets of images (two reference and four harmonic), each set comprising of a sequence of 29 images each with a FOV of $320 \mathrm{~mm}$. These images were acquired in just six seconds. The data sets were upsampled to a matrix size of $512 \times 512$, 


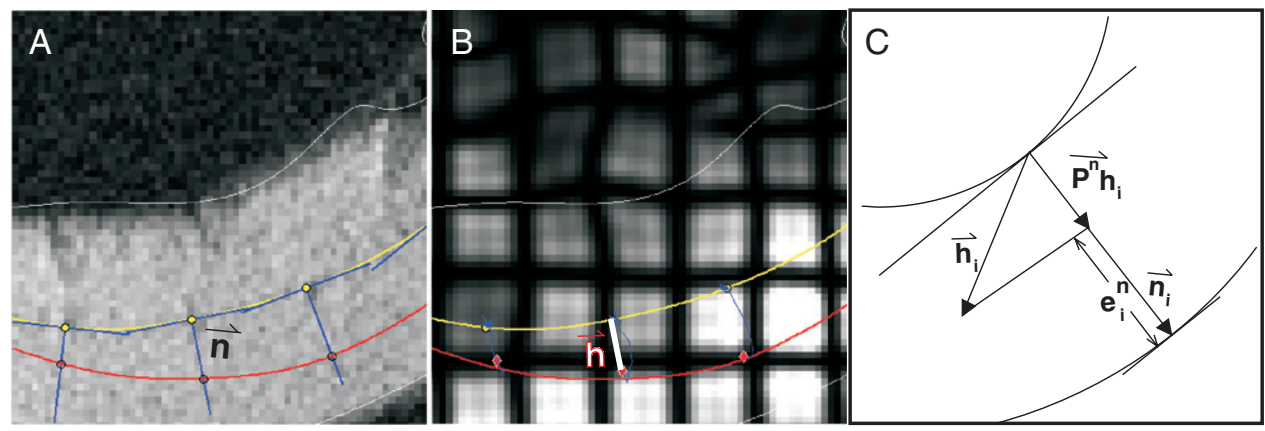

Fig. 6. (A) Zoomed region of high-resolution image at 391 ms: yellow line, Mesh1; red line: Mesh2. Yellow dots indicate points selected on Mesh1; blue lines, normal and tangent lines drawn at each point; black circles, points of intersection of Mesh2 with the normal lines. (B) Zoomed region of a synthetic tagged image at $394 \mathrm{~ms}$. Red diamonds indicate HARP tracked points at $1296 \mathrm{~ms}$; blue lines, tracked trajectories from 394 to 1296 ms. (C) Diagrammatic representation of the normal error in the 2-D motion calculated for each selected MR marker.

and SF-harmonic images for the two orthogonal tagging directions [described in Eqs. (16) and (17)] were generated.

\subsubsection{Tracking errors}

To determine the reliability of the 2-D HARP tracking results obtained from these SF-harmonic images, a simple experiment was conducted on a moving phantom. A set of selected points lying on a mesh made of elastic netting that was inserted in the phantom were tracked using HARP 2-D tracking on data acquired using the SF-HARP MRI pulse sequence and compared to an independent truth model obtained using the high-resolution data set.

As seen in Fig. 5(A) and (B), light traces of the mesh and air bubbles near it are visible in the high-resolution images obtained during the first scan. From the high-resolution images, mesh boundaries were determined by manually selecting points on visible landmarks, and spline interpolation was used to get a densely sampled curve representation. The black solid lines in Fig. 5(A) and (B) illustrate the mesh boundaries at two time frames: $391 \mathrm{~ms}$ for Mesh1 and $1292 \mathrm{~ms}$ for Mesh2. At the first of these two time frames, the gel phantom was in its normal state, while at the second, it was at its maximum state of expansion. The through-plane motion in this phantom experiment was very small; hence, these mesh boundaries generated were assumed to be the truth model of the 2-D motion. Outer and inner boundary curves (white lines) are superimposed for visual reference.

Next, from the reconstructed SF-harmonic images, gridtagged images [33] at two very close times - 394 and 1296 $\mathrm{ms}$ - were generated by performing a simple Fourier series expansion on the combined harmonic phase obtained from Eq. (17) and are illustrated in Fig. 5(C) and (D). Note the straightening or stretching of the tag lines in Fig. 5(D), corroborating the gel expansion at $1296 \mathrm{~ms}$. The tag lines outside the external border and within the internal border of the phantom is due to tagged rubber sheet lining used in the phantom construction.

Thirty points were arbitrarily selected on Mesh1 [gray dots on the black solid line in Fig. 5(A)], and the 2-D motions of these points were determined using two independent schemes. First, for the high-resolution images, the two curves were superimposed on the same image as shown in a zoomed region in Fig. 6(A).

At each point, the equations of the tangent and normal lines to Mesh1 (yellow solid line) were determined and drawn [blue lines in Fig. 6(A)]. The distance between the selected point (yellow dot) and the point of intersection of the normal with Mesh2 (black circles on the red solid line) was referred to as the normal tracked distance. Second, for the SF-HARP reconstructed images, the set of selected points on Mesh1 were tracked using 2-D HARP tracking tools, and the 2-D motion trajectories from 394 to $1296 \mathrm{~ms}$ were obtained as illustrated by the blue lines in Fig. 6(B). The red diamonds depict the final position of the points at $1296 \mathrm{~ms}$. The two meshes are superimposed for purposes of comparison. Next, for each point, the harp tracked vector $\mathbf{h}_{i}$, delineated in Fig. 6(C), was projected onto the normal vector $\mathbf{n}_{i}$ to give $\mathbf{P}^{n} \mathbf{h}_{i}$, and an error measure comparing the harp tracked distance to the normal tracked distance was computed as $e_{i}^{n}=\left|\mathbf{P}^{n} \mathbf{h}_{i}-\mathbf{n}_{i}\right|$.

\subsection{Experimental methods: in vivo studies}

\subsubsection{Image acquisition}

All in vivo studies were approved by the institutional review board of Johns Hopkins University, and informed consent was obtained from all subjects. Experiments were conducted on three normal volunteers of age 25-28 years and a heart rate of $60-75$ beats per minute with no prior history of cardiac disease or chest pain. Representative data from one normal volunteer is emphasized in this article. In this volunteer experiment, eight SA slices and six LA slices were prescribed, each with an FOV of $34 \mathrm{~cm}$. The SF-HARP MRI pulse sequence with imaging parameters described above was used to acquire these slices in four short breathhold scans. During the first (resp. second) 20-s breath-hold, four apical to mid (resp. mid to basal) SA slices were acquired sequentially. During the third (resp. fourth) 15 -s breath-hold, three LA slices oriented at $0^{\circ}, 30^{\circ}$ and $60^{\circ}$ (resp. $90^{\circ}, 120^{\circ}$ and $150^{\circ}$ ) were acquired sequentially.

For each slice, six data sets (two reference and four harmonic) were acquired in six heartbeats. Each data set 


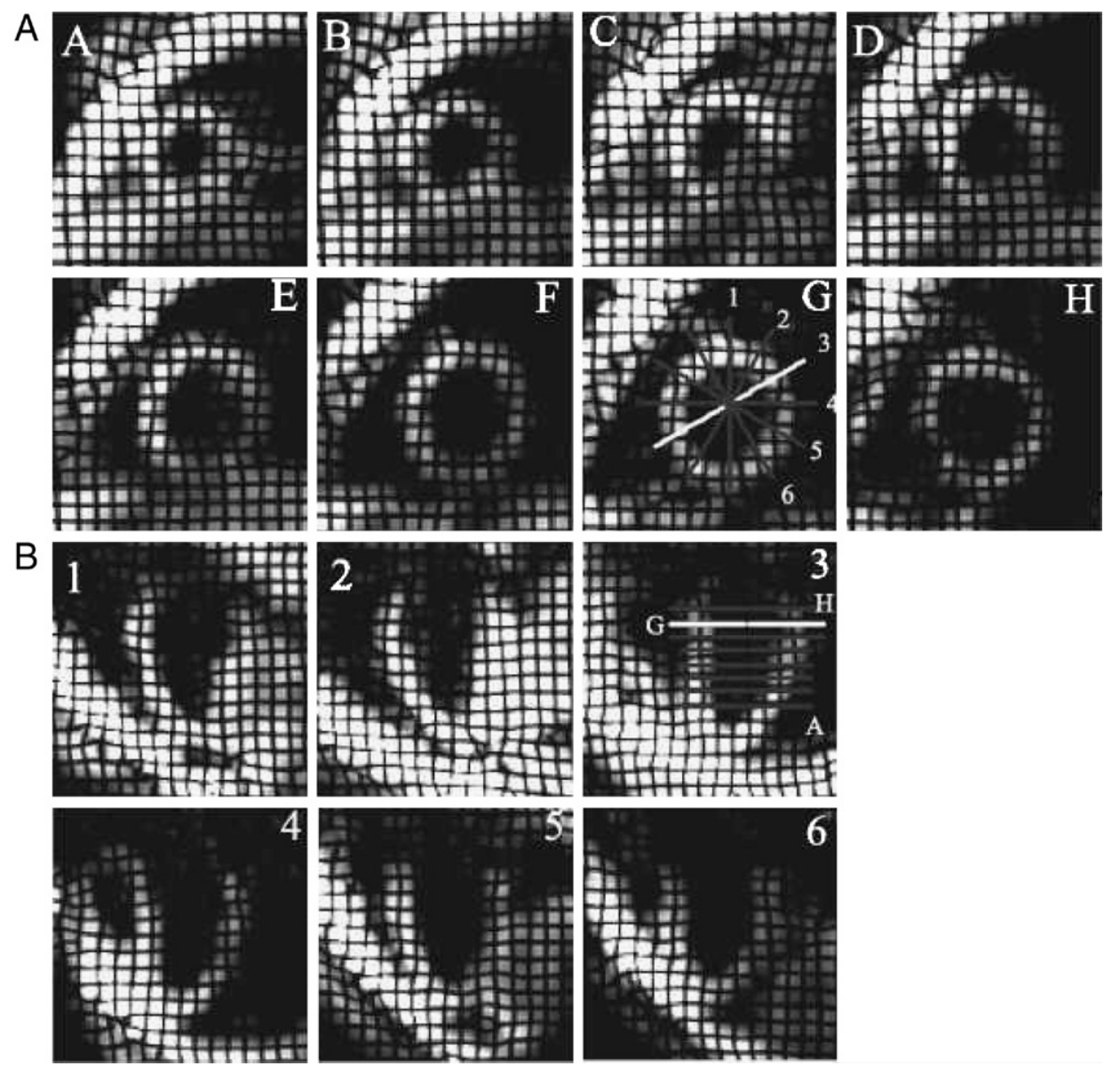

Fig. 7. Representative synthetically grid-tagged images obtained using the SF-HARP MRI pulse sequence. (A-H) Eight SA slices displayed from apex to base. (1-6) Six LA slices acquired. White dots indicate grid points lying on the line of intersection of SA plane G and LA plane 3; blue dots: all other grid points selected on SA plane G and LA plane 3.

comprised twelve images that were upsampled to size $256 \times$ 256 , individually reconstructed and combined to generate a sequence of SF harmonic images [see Eqs. (16) and (17)] over $85 \%$ of the R-R cycle for each orthogonal tagging direction in every slice. Representative tagged images generated from the SF-harmonic images at a diastolic time frame for each of the SA and LA slices are depicted in Fig. 7(A) and (B), respectively.

The SA slices are marked with letters A-H from the most apical to the most basal slice, and the LA slices are marked with numbers $1-6$ beginning with a slice oriented in the anterior posterior direction and moving clockwise with increments of $30^{\circ}$.

\subsubsection{MR markers}

To identify a set of MR markers, lines of intersections of each SA and LA tag planes were first determined from the image plane coordinates of the acquired tag planes, and a regular grid of points, emanating from the center, on each of these lines of intersections were defined automatically on an end-diastolic reference time frame. The grid points for one SA image are displayed in Fig. 7(A), panel G, and those for one LA image are displayed in Fig. 7(B), panel 3. Next, for each SA-LA plane intersection, the corresponding grid points were tracked on each 2-D plane using standard HARP tracking algorithms and points outside the myocardium were manually removed. (This is the only manual step that was used besides slice prescription; this step is not

A

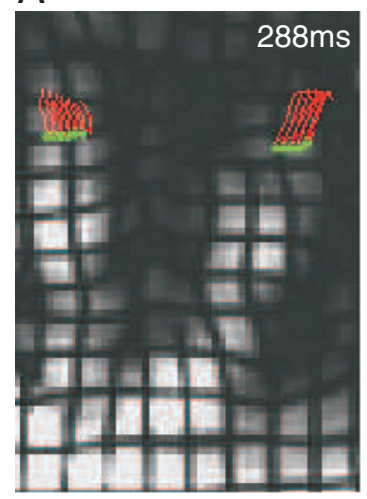

B

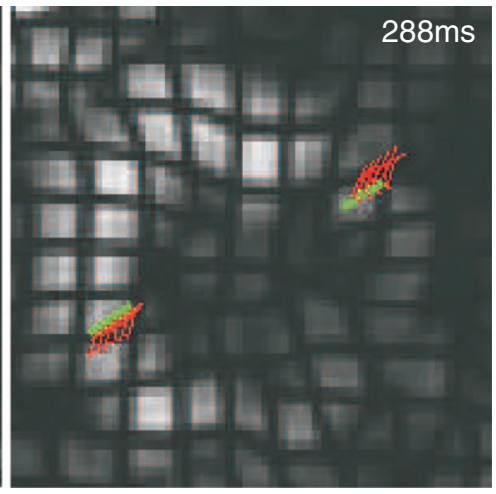

Fig. 8. End-systolic zoomed images of (A) LA slice 5 and (B) SA plane G. Red traces represent the 2-D tracks of a group of gridpoints lying within the myocardium. Green dots represent the position of the points at the endsystolic time frame. 


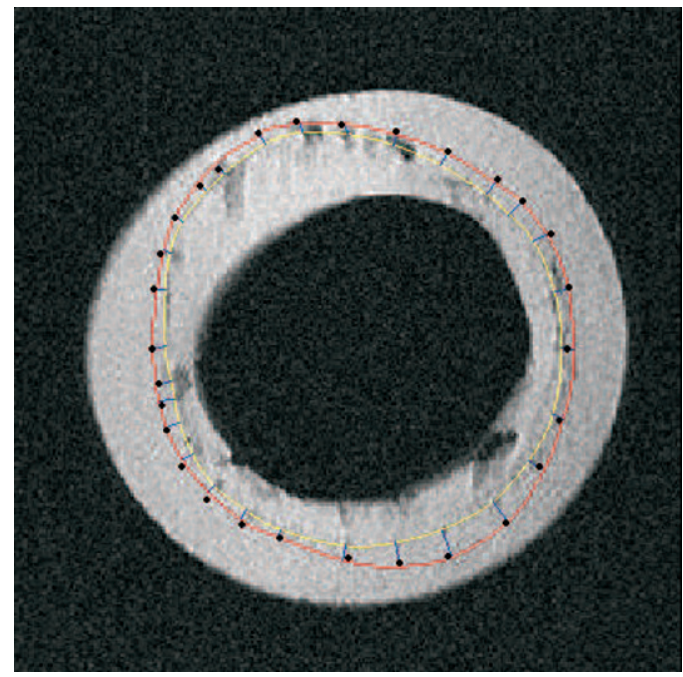

Fig. 9. High-resolution image at $391 \mathrm{~ms}$ with superimposed Mesh1 (yellow line) and Mesh2 (red line). Blue lines represent normal tracked distances from 391 to $1292 \mathrm{~ms}$, and black dots represent the HARP tracked points at $1296 \mathrm{~ms}$.

strictly necessary but it is convenient since it restricts the tracked points to the myocardium.) A view-sharing reconstruction scheme was employed to obtain interpolated tracking measurements every $12 \mathrm{~ms}$. The SA and LA trajectories are displayed as red traces in zoomed regions of image planes $\mathrm{G}$ and 3 at an end-systolic time frame in Fig. 8 . The green dots in the figure represent the position of the MR marker at the time frame in view. Finally, the SA and LA trajectories were combined to obtain a collection of MR marker 3-D pathlines.

\subsubsection{Global measures}

Three different global measures of LV motion were obtained from the marker trajectories: (1) evolution of the rotation angle averaged over an apical, a midventricular, and a basal slice; (2) evolution of midwall circumferential strains averaged over four regions (anterior, posterior, lateral and septal) for a midventricular slice and (3) evolution of the average base-apex longitudinal strain. These measurements were all computed with respect to a reference time frame, which we selected to be the first time frame in the sequence of images. For every marker located on a selected SA slice, the rotation angle at any time $t$ was defined as the angle between the radial line connecting the center of the LV cavity to the marker position at the reference time frame and the radial line connecting the center of the LV cavity to the marker position at time $t$. The sign of the rotation angle determines the marker position relative to its position at the reference time frame; movement in the counterclockwise direction is considered positive, while movement in the clockwise direction is considered negative. The average rotation angle for all markers selected on one basal, one midventricular and one apical slice was then computed. To measure circumferential shortening, one marker was selected along each radial line in the midwall on each slice. The

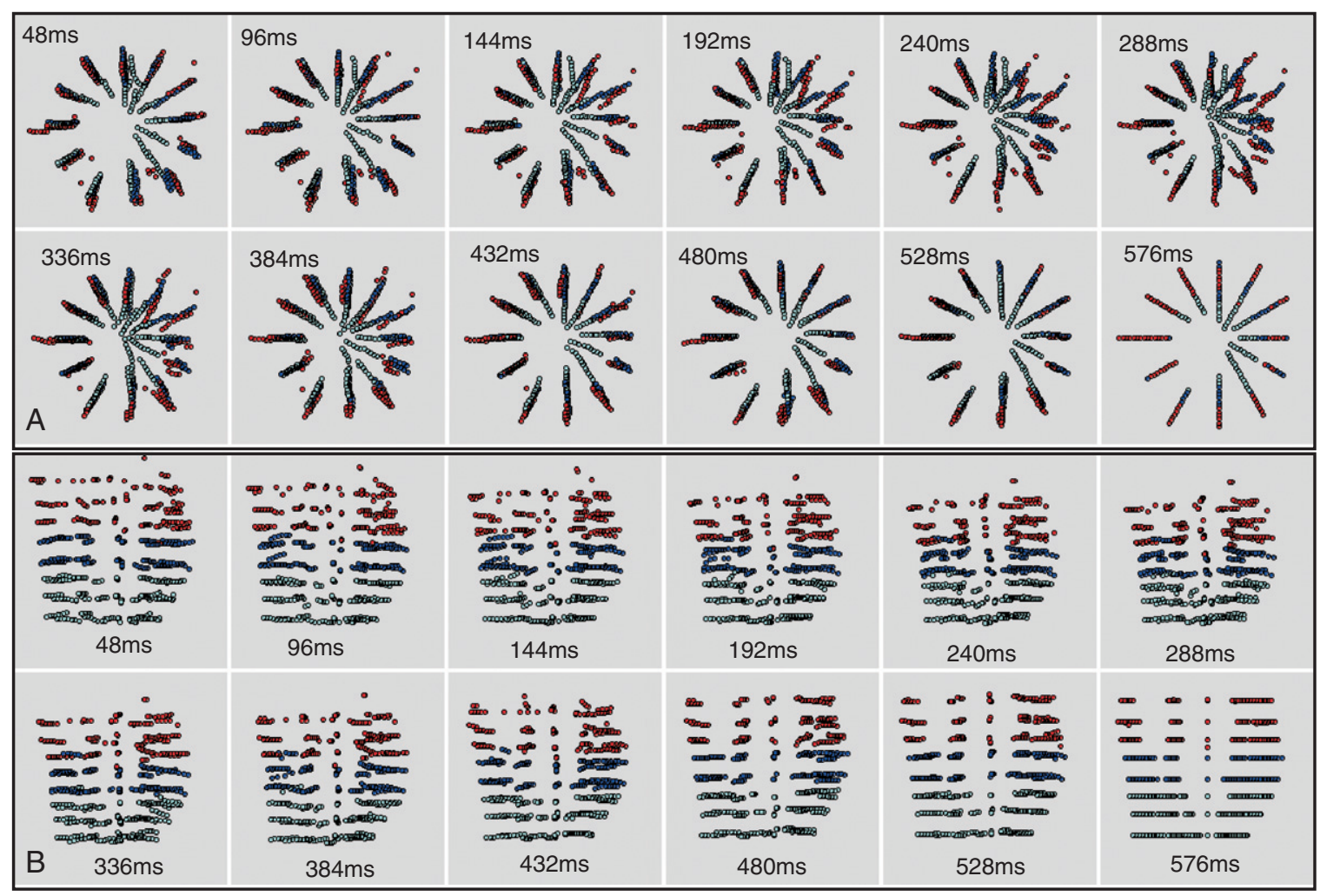

Fig. 10. All SA-LA slice intersection grid point locations at 12 time frames for (A) an SA view looking down from the base to the apex and (B) an LA view looking sideward with the free wall on the right. 
percent change in the distance between every set of two adjacent markers at any time $t$ with respect to the reference time frame was defined as the midwall circumferential strain. The average circumferential strain computed in four regions defined in the heart was then computed. Finally, the percentage change in the average longitudinal distance between any two selected slices with respect to the reference time frame $t$ was defined as the average longitudinal compression between those slices.

\section{Results}

\subsection{Phantom study}

Results from the phantom study are shown in Fig. 9. The figure displays both the normal tracked results (short blue lines) and the HARP tracked points (black dots) on the highresolution phantom image at $391 \mathrm{~ms}$. Mesh1 (yellow solid line) and Mesh2 (red solid line) are also superimposed on the image. It can be observed that the HARP tracked points desirably lie on or very close to Mesh2. In addition, the root mean square percentage error for the thirty selected points was found to be $12 \%$. These results demonstrate comparable 2-D motion estimates of a set of points in the moving phantom using two independent measurement schemes.

\subsection{In vivo studies}

Fig. 10 shows the 3-D locations of all the MR markers from all slices at all time frames in two different orientations demonstrating the 3-D nature of left ventricular cardiac motion. For better visualization and analysis, the MR markers are color-coded. Markers lying on (1) the three most apical slices A-C are colored in cyan, (2) the two midventricular slices D and $\mathrm{E}$ are colored in blue, and (3) the three most basal slices $\mathrm{F}-\mathrm{H}$ are colored in red. From these images, there is evidence of end-systolic radial thickening, circumferential shortening, and longitudinal compression. We also notice an anticlockwise twist of the points during systole in the apical slice and a corresponding clockwise untwisting motion during diastole. The markers on the basal slice show a slight twisting motion but in the reverse direction to the apical slice.

Fig. 11(A) displays the evolution of the average rotation angles in degrees as a function of time in ms computed on the apical slice B (solid line with dots), the midventricular slice D (dot-dashed line with circles) and the basal slice G (dotted line with crosses). For the apical slice, we observe a counterclockwise systolic twist followed by a clockwise diastolic twist, with a maximum systolic twist angle of $6.8^{\circ}$ at end-systole. The midventricular slice behaves in a similar fashion achieving a peak systolic rotation angle of $2^{\circ}$. The basal slice, however, twists counterclockwise in early systole and then rotates clockwise during the rest of systole and counterclockwise during diastole, producing a maximum negative rotation of $5.5^{\circ}$ at end-systole. It is well known that the diastolic untwisting motion begins with an early rapid recoil with little change in LV volume, followed by the diastolic suction during which the bulk of the diastolic filling occurs. We notice that this middiastolic expansion is preceded with a slight twist in motion. This is
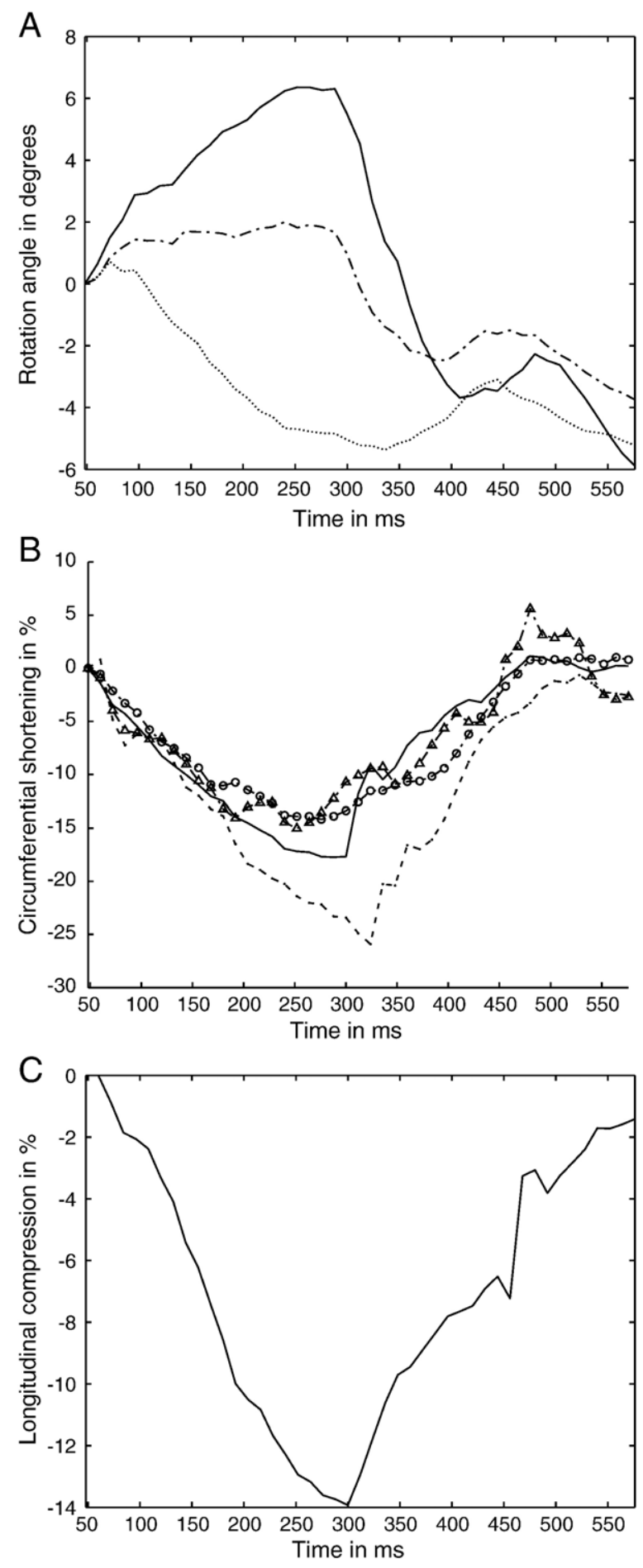

Fig. 11. (A) Evolution of average rotation angle in degrees for apical slice B (solid line with dots), midventricular slice D (dot-dashed line with circles), and basal slice $\mathrm{G}$ (dotted line with crosses). Evolution of average (B) circumferential strain and (C) evolution of average longitudinal strain. 
captured in the bump observed in the rotation angle curves at mid diastole (around 375-425 ms).

Fig. 11(B) shows the evolution of the average midwall circumferential shortening in the lateral, septal, anterior and posterior regions of the heart. These curves show greater shortening in the free wall with peak average lateral circumferential shortening of about $22 \%$. The average circumferential shortening in all other regions is lower, and the peak systolic shortening is in the range of $13-15 \%$.

Fig. 11(C) shows the evolution of the average longitudinal strains indicating a maximum end-systolic longitudinal compression of $12.5 \%$. Fig. 10(B) shows that this longitudinal compression occurs geometrically by a large downward basal motion together with a slight upward apical motion. Similar trends in these results were observed in the other two normal volunteer experiments. From all three volunteers, peak systolic average longitudinal compressions of $13.16 \% \pm 0.76 \%$ (mean \pm S.D.), peak systolic average lateral midwall circumferential strains of $23.5 \% \pm 1.5 \%$ (mean \pm S.D.), and peak systolic average apical rotations of $6.5^{\circ} \pm 0.76^{\circ}$ (mean \pm S.D. $)$ were obtained.

\section{Discussion}

In this article, we demonstrated an automatic method that can perform true 3-D tracking of material points lying on lines of intersections of orthogonal 2-D tagged image planes. The density of these tracked lines depends on the number and spacing of the image planes; there is a direct relationship between the number of lines in space that can be tracked and the image acquisition time. The implementation of the method in a fast imaging framework adds to the novelty and potential utility of the overall scheme. The image acquisition protocol can be as short as eight cardiac cycles, which is sufficient to track a single line or, as long as it is feasible, a few short breath-holds in practice in order to track a few dozen lines throughout the myocardium.

In comparison to existing tag-based point tracking techniques [18,20,21,23,24,34], our method provides direct and fast measurements of 3-D motion with minimal (or no) user interaction. In phase-contrast tracking techniques $[3,4]$, the integration of the velocity data introduces high timeresolution demands to achieve a good estimation of the 3-D motion trajectory. This can result in time-consuming scans that are cumbersome to perform. Using SF-HARP, the 2-D motion trajectories are directly obtained by tracking the 2-D phase vectors without an additional nonlinear operation performed on the phase function.

The high speed of acquisition using HARP MRI results in a loss of spatial resolution compared to existing phasecontrast [5] or stimulated echo-based techniques [6,7]. This makes our technique sensitive to regional and global abnormalities rather than localized small dysfunctions. Employing high-resolution slice following tagged image acquisitions in conjunction with the "cosine and sine modulation to eliminate" approach [35] will enable isolation of harmonic peaks using large-sized HARP filters generating high-resolution SF-HARP tracking results but at the cost of imaging time.

As compared to standard harmonic images, SF-harmonic images demonstrate a $10-15 \%$ decrease in signal-tonoise ratio (SNR) for two main reasons. First, the nuclear spins in the moving material slice are repeatedly excited by imaging flip angles, which decreases the relaxation times during SF-HARP MRI. When SF is not used, as in HARP MRI, there is through-plane motion of the tissue in and out of the imaged slab. Depending on the amount of throughplane motion, a small or large portion of the nuclear spins in the imaged slice is not repeatedly excited by every imaging flip angle applied. This results in a decreased overall transverse decay of the bulk magnetization in the fixed slice and hence improved SNR. Second, there is a much larger DC spectral peak in SF-harmonic images due to the thicker slices imaged. Improper CSPAMM subtraction results in an increased presence of unwanted interference signals subsequently decreasing the SNR in the SF-harmonic images. The legitimacy of 2-D point tracking on these reduced-SNR SF-HARP data sets was demonstrated in the phantom experiment. Although the results from this experiment are preliminary in nature, they provide an indication of the reliability in the tracking results using this technique, especially when a smooth continuous motion is being measured.

The in vivo experimental results in the normal volunteers demonstrate typical characteristics of longitudinal compression, circumferential shortening, radial thickening and apical rotation. As in previous practice (e.g., Ref. [34]), we elected to continue displaying the MR markers as tiny spherical beads. The two-orientation view was used, as it provided a complete understanding of all the major modes of 3-D left ventricular motions. In our normal volunteer experiments, we chose a constant imaging slice thickness of $30 \mathrm{~mm}$ with coincident tag slice and imaging slice centers. For a couple of basal slices, we find that this slice thickness was not large enough to encompass the tagged slice completely during end-systole, especially in regions of the free wall. This yields a lessening of tissue signal in these regions, a resulting increase in computed phase errors, and erroneous tracked pathlines, which are evident as stray red dots in Fig. 10. In the future, the center position of the tagged slice within the image slab can be adaptively moved closer to the top of the image slab as more basal slices are imaged, allowing for a greater downward motion in these slices. The HARP 2-D tracking algorithm works on wrapped phase images with the inherent assumption that the motion of any given point from one image to the next does not exceed the tag period. This assumption, however, fails in some basal free wall regions in LA slices due to the rapid untwisting during the early mid-diastolic phase. In this experiment, we manually corrected the tracking trajectories. In the future, this process should be automated. 


\section{Acknowledgment}

The authors are extremely grateful to Andy Derbyshire for his guidance in pulse sequence programming.

\section{References}

[1] Zerhouni EA, Parish DM, Rogers WJ, Yang A, Shapiro EP. Human heart: tagging with MR imaging — a method for noninvasive assessment of myocardial motion. Radiology 1988;169:59-63.

[2] Axel L, Dougherty L. MR imaging of motion with spatial modulation of magnetization. Radiology 1989;171:841-5.

[3] Pelc NJ, Herfkens RJ, Shimakawa A, Enzmann D. Phase contrast cine magnetic resonance imaging. Magn Reson Q 1991;7(4):229-54.

[4] Constable RT, Rath KM, Sinusas AJ, Gore JC. Development and evaluation of tracking algorithms for cardiac wall motion analysis using phase velocity MR imaging. Magn Reson Med 1994;32:33-42.

[5] Reese TG, Feinberg DA, Dou J, Wedeen VJ. Phase contrast MRI of myocardial 3-D strain by encoding contiguous slices in a single shot. Magn Reson Med 2002;47:665-76.

[6] Aletras AH, Ding S, Balaban RS, Wen H. DENSE: displacement encoding with stimulated echoes in cardiac functional MRI. J Magn Reson Imaging 1999;137:247-52.

[7] Wen H, Pai V. Mapping 3D myocardial wall motion in the left ventricle over a breath-hold. Proceedings of the International Symposium on Biomedical Imaging. Washington, DC: IEEE-EMBS; 2002. p. 121-4.

[8] Maier SE, Fischer SE, McKinnon GC, Hess OM, Krayenbuehl HP, Boesiger P. Evaluation of left ventricular segmental wall motion in hypertrophic cardiomyopathy with myocardial tagging. Circulation 1992;86:1919-28.

[9] Sigwart CJ, Heintzen PH. Ventricular wall motion. New York: Thieme-Stratton; 1984.

[10] Liberman AN, Weiss JL, Jugdutt BI, Becker LC, Weisfeldt ML. Twodimensional echocardiography and infarct size: relationship of regional wall motion and thickening to the extent of myocardial infarction in the dog. Circulation 1981;63:739-46.

[11] Nagel E, Stuber M, Lakatos M, Scheidegger MB, Boesiger P, Hess OM. Cardiac rotation and relaxation after anterolateral myocardiac infarction. Coron Artery Dis 2000;11(3):261-7.

[12] Nagel E, Stuber M, Lakatos M, Hess OH, Boesiger P. Loss of cardiac rotation and diastolic untwisting after anterolateral myocardial infarction. Proceedings of the International Society of Magnetic Resonance in Medicine. Hoboken (NJ): John Wiley \& Sons; 1996.

[13] Buchalter MB, Sims C, Dixon AK, Weissberg PL, Stone DL, Shah NJ, et al. Measurement of regional left ventricular function using labelled magnetic resonance imaging. Br J Radiol 1991;64:953-8.

[14] Stuber M, Nagel E, Fischer SE, Steinemann F, Boesiger P. Assessment of systolic and diastolic ventricular torsion in pathologic and physiologic hypertrophied hearts. Proceedings of the International Society of Magnetic Resonance in Medicine. Hoboken (NJ): John Wiley \& Sons; 1996.

[15] Matter C, Nagel E, Stuber M, Boesiger P, Hess OM. Assessment of systolic and diastolic LV function by MR myocardial tagging. Basic Res Cardiol 1996;91:23-8.

[16] McVeigh ER, Prinzen FW, Wyman BT, Tsitlik JE, Halperin HR, Hunter WC. Imaging asynchronous mechanical activation of the paced heart with tagged MRI. Magn Reson Med 1998;39: 507-13.

[17] Amini AA, Prince JL. Measurement of cardiac deformations from MRI: physical and mathematical models. Dordrecht: Kluwer Academic Publishers; 2001.

[18] Guttman MA, Prince JL, McVeigh ER. Tag and contour detection in tagged MR images of the left ventricle. IEEE Trans Med Imaging 1994;13(1):74-88.

[19] Ryf S, Spiegel MA, Gerber M, Boesiger P. Myocardial tagging with 3D-CSPAMM. J Magn Reson Imaging 2002;16(3):320-5.

[20] O'Dell WG, Moore CC, McVeigh ER. Displacement field fitting approach to calculate 3D deformations from parallel-tagged $\mathrm{MR}$ images. J Magn Reson Imaging 1993;3(P):208.

[21] Young AA, Kraitchman DL, Dougherty L, Axel L. Tracking and finite element analysis of stripe deformation in magnetic resonance tagging. IEEE Trans Med Imaging 1995;14(3):413-21.

[22] Young AA, Kramer CM, Ferrari VA, Axel L, Reichek N. Threedimensional left ventricular deformation in hypertrophic cardiomyopathy. Circulation 1994;90(2):854-67.

[23] Moulton MJ, Creswell LL, Downing SW, Actis RL, Szabo BA, Vannier MW, et al. Spline surface interpolation for calculating 3-D ventricular strains from MRI tissue tagging. Am J Physiol 1996;270(Heart Circ. Physiol. 39):H281-97.

[24] Amini AA, Chen Y, Curwen RW, Mani V, Sun J. Coupled B-snake grids and constrained thin-plate splines for analysis of 2-D tissue deformations from tagged MRI. IEEE Trans Med Imaging 1998;17(3):344-56.

[25] Osman NF, McVeigh ER, Prince JL. Imaging heart motion using harmonic phase MRI. IEEE Trans Med Imaging 2000;19: 186-202.

[26] Osman NF, Prince JL. Visualizing myocardial function using HARP MRI. Phys Med Biol 2000;45:1665-82.

[27] Fischer SE, McKinnon GC, Maier SE, Boesiger P. Improved myocardial tagging contrast. Magn Reson Med 1993;30:191-200.

[28] Fischer SE, McKinnon GC, Scheidegger MB, Prins W, Meier D, Boesiger P. True myocardial motion tracking. Magn Reson Med 1994;31:401-13.

[29] Stuber M, Fischer SE, Scheidegger MB, Boesiger P. Toward highresolution myocardial tagging. Magn Reson Med 1999;41:639-43.

[30] Sampath S, Derbyshire JA, Osman NF, Atalar EA, Prince JL. Realtime imaging of cardiac strain using ultra fastHARP sequence. Proc. International Society for Magnetic Resonance in Medicine. Hoboken (NJ): John Wiley \& Sons; 2001. p. 111.

[31] Bernstein MA, Grgic M, Brosnan TJ, Pelc NJ. Reconstructions of phase contrast, phased array multi coil data. Magn Reson Med 1994;32:330-4.

[32] Sampath S, Parthasarathy V, Prince JL. A phantom validation of the fastHARP pulse sequence. Proc. International Symposium on Biomedical Imaging, Washington, DC; 2002.

[33] Osman NF, Sampath S, Derbyshire JA, Atalar E, Prince JL. Synthetic tagged MR images for real-time HARP imaging. Proc. of the 9th Annual Meeting of ISMRM; 2001.

[34] Kerwin WS, Prince JL. Cardiac material markers from tagged MR images. Med Image Anal 1998;2(4):339-53.

[35] Epstein FH, Gilson WD. Displacement-encoded cardiac MRI using cosine and sine modulation to eliminate (CANSEL) artifact-generating echoes. Magn Reson Med 2004;52:774-81. 